\title{
Vasomotor Effect after Acute Intoxication with Bupivacaine and Levobupivacaine in Rats Via Intraperitoneal Route Analyzed via Digital Infrared Imaging
}

\author{
Angelo Manoel Grande Carstens ${ }^{1}$, Elizabeth Milla Tambara, TSA 2, Jorge Eduardo Fouto Matias ${ }^{3}$, \\ Marcos Leal Brioschi ${ }^{4}$, Daniel Colman ${ }^{5}$, Márcio Grande Carstens ${ }^{6}$
}

Summary: Carstens AMG, Tambara EM, Matias JEF, Brioschi ML, Colman D, Carstens MC - Vasomotor Effect after Acute Intoxication with Bupivacaine and Levobupivacaine in Rats Via Intraperitoneal Route Analyzed via Digital Infrared Imaging.

Background and objectives: The study of the vasomotor effect of local anesthetics (LA) is of paramount importance for the analysis of the occurrence of cardiotoxic and neurotoxic effects, and drug interactions. In order to find a safer drug than racemic bupivacaine, this study aimed to analyze digital infrared imaging of acute vasomotor effect of bupivacaine and levobupivacaine in rats intraperitoneally.

Method: We used 30 male Wistar rats distributed into three groups $(n=10)$ and subjected to an intraperitoneal injection of LA. In Group C (control) $1 \mathrm{~mL} 0.9 \%$ saline was injected intraperitoneally. In Group B (bupivacaine), intraperitoneal injection of $0.5 \%$ of racemic bupivacaine (S50-R50), dose of 20 mg. kg-1 of body weight. In Group L (levobupivacaine), intraperitoneal injection of levobupivacaine $0.5 \%$ enantiomeric excess (S75-R25) in dose of $20 \mathrm{mg}^{\mathrm{kg}}{ }^{-1}$ of body weight. The procedure was thermographicly continuously filmed from the time of pre-injection until 30 minutes after injection. The results of the recordings were analyzed in graphical form, verifying the maximum temperature of each rat and the average temperature of the system that housed the animal.

Results: The results of graphic analysis showed no difference between Group L and Group C, and the average temperature remained stable throughout the experiment in both groups. In Group B, there was a phenomenon of temperature increase after intraperitoneal injection of bupivacaine.

Conclusions: The results demonstrated that the vasomotor effect of the acute toxicity of levobupivacaine was similar to Group $\mathrm{C}$ with saline, through macroscopic studies by infrared digital filmmaking, and that there were vasomotor changes (vasoconstriction), with bupivacaine intoxication in relation to both Group C and Group L.

Keywords: Bupivacaine, levobupivacaine; Vasoconstriction; Thermography; Rats.

[Rev Bras Anestesiol 2011;61(2): 188-201] @Elsevier Editora Ltda.

\section{INTRODUCTION}

Clinical activity of local anesthetics (LA) varies according to their structure and can be modulated by many factors, physicochemical nature, such as lipid solubility, pKa, percentage of protein binding. The application of stereoisomerism in the pharmaceutical industry enabled the emergence of local anesthetics increasingly elaborated ${ }^{2}$.

Bupivacaine is one of the most commonly used LA in blocks, because of its potency related to lipid solubility, the sensory

Received from Universidade Federal do Paraná (UFPR), Paraná, Brazil.

1. Anesthesiologist Post-Graduate in Occupational Health, M.Sc. in Surgery, UFPR, Ph.D. Student, Graduate Program in Clinical Surgery, UFPR

2. Chief Professor in the Department of Anesthesiology, PUC-PR, Associate Professor in the Department of Anesthesiology, UFPR; Ph.D. in Clinical Surgery, UFPR

3. Associate Professor, Department of Surgery, UFPR; Ph.D. in Digestive Surgery at the University of Montpellier, France

4. Visiting Professor at the Graduate Department of Surgery UFPR; Medical Researcher, Center for Pain HCFMUSP, Ph.D., Department of Mechanical Engineering UFPR; Post-doc at the Department of Neurology - Pain Center HCFMUSP.

5. Anesthesiologist and Medical Examiner, PhD in Clinical Surgery, UFPR

6. ME3 CET / SBA do Hospital de Clínicas, UFPR.

Submitted on February 27, 2010

Approved on October 23, 2010.

Correspondence to:

Dr. Angelo Manoel G. Carstens

Rua Coronel Menna Barreto Monclaro,510

Centro

80420-070 - Curitiba, PR, Brazil

E-mail: angelocarstens@pop.com.br block of excellent quality and the absence of pronounced motor block by average concentration $(0.5 \%)$. Its therapeutic index is small, because, unlike lidocaine, the differences in cardiotoxic and neurotoxic concentrations are very low, so that a cardiotoxic accident may occur without neurological prodrome, according to some reports of unexpected accidents with their clinical use ${ }^{3}$. Bupivacaine is an equimolar mixture of two enantiomers: $\mathrm{R}(+)$ and $\mathrm{S}(-)$ bupivacaine. Starting from purified isomers of local anesthetic molecule, it is possible to manipulate the enantiomeric ratio of a racemic compound. This might contribute to their effectiveness and decrease potential toxicity, with increased therapeutic index. Levobupivacaine is constituted by enantiomeric mixture of bupivacaine (S75-R25) $0.5 \% 4$. Levobupivacaine is less toxic if compared to bupivacaine ${ }^{5-7}$, but there are also reports of accidents with levobupivacaine ${ }^{8}$.

The effects of bupivacaine toxicity in peripheral vascular resistance are controversial, being reported both vasodilation and vasoconstriction. A study successfully tested the hypothesis that the use of vasodilators allows lower neurological toxicity in a rat model subjected to continuous infusion of bupivacaine ${ }^{9}$. It is suggested that vasoconstrictors accentuate the neurotoxicity by increased plasma concentration of bupivacaine and, perhaps, vasodilators have a protective effect. Other studies were conducted with the use of vasoconstrictors ${ }^{10}$ and demonstrated that the increased peripheral vasoconstriction significantly increases the toxicity, requiring 
lower doses of LA for seizures and cardiac arrest. The use of vasodilators associated with LA, as nicardipine and phentolamine resulted in a protective effect on acute venous LA ${ }^{11}$. Several other studies were conducted with the combination of vasoconstrictors, vasodilators, intravenous or inhalational anesthetics, showing controversial results on the study of the toxicity of local anesthetic ${ }^{12,13 .}$

Interactions with other drugs are probably more common than expected and can be both beneficial and harmful. Also, the hemodynamic effects of local anesthetics are offered in four anatomical sites: direct action on cardiac or vascular smooth muscle; direct action on the autonomic nerves, evoked reflexes; direct action in the CNS ${ }^{14}$.

Adding to the wide range of variables that can alter the vasomotor and, moreover, the possible interactions between drugs, the creation of a study model is justified in order to comparatively evaluate the vasomotor effect of the acute toxicity of bupivacaine as compared to levobupivacaine intraperitoneally in rats, using infrared thermography.

\section{METHODS}

The experimental protocol used in this study was conducted according to ethical principles of the Brazilian College of Animal Experimentation (COBEA, 1999). The experimental protocol was approved by the Ethics Committee of Hospital de Clínicas, UFPR.

We used 30 male Wistar rats (Rattus norvegicus albinos, Rodentia mammalia), aged between 120 and 153 days (mean 135.9 days). Preoperatively, a standard ration and water ad libitum are provided until 12 hours before the procedure.

The experiment was conducted at the Laboratory for Research in Postgraduate Surgical Clinic UFPR. A minimum thermal variation with ambient temperature was assumed, with environmental temperature maintained at $21^{\circ} \mathrm{C}$ and relative humidity at $75 \%$. Measurements were checked with dry and wet bulb thermo-hygrometer (Incotherm, Br). Heat loss by convection was minimized by keeping doors and windows closed and minimal movement around the animals. The airflow was controlled with a digital anemometer with rotation blades model HHF 300 A (Omega Engineering, Inc), a distance of $10 \mathrm{~cm}$ from the animal, keeping the velocity of air flow less than $0.2 \mathrm{~ms}^{-1}$. This is the value of transition between heat loss by free and forced convection.

The animals were distributed into three groups:

Group C - Control: intraperitoneal injection of saline $1 \mathrm{~mL} 0.9 \%$ Group B - intraperitoneal injection of bupivacaine $0.5 \%$ at 20 mg.kg-1

Group L - intraperitoneal injection of levobupivacaine $0.5 \%$ at $20 \mathrm{mg} \cdot \mathrm{kg}^{-1}$

The animals were placed in cages with five animals each. At the time of the experiment, one animal was allocated to a single cage. Once free of environmental interference with anesthesia and other drugs, thermographic continuous filming was performed at three times:
- Pre-injection continuously documented by the imager;

- Intraperitoneal injection of local anesthetic, according to the groups;

- Observation and filming of the vasomotor effects of the drug injected intraperitoneally for a period of 30 minutes.

We used a sensor ThermoVision AGEMA550 (FLIR SystemsTM, Sweden) to capture the spectral range of electromagnetic waves emitted between 3.5 to $5 \mu \mathrm{m}$ (waves in the middle infrared spectrum). Maximum spatial resolution obtained was 0.1 to $0.2 \mathrm{~mm}$.

The infrared radiation naturally emitted by objects in the environment is captured and converted into an electrical signal through a PTSI detector, cooled with liquid nitrogen (steerling cycle). This signal is processed in a spreadsheet number of 76,000 points of absolute temperature calibrated by frame, represented instantly on the thermal image with a resolution of $320 \times 240$ pixels and thermal sensitivity greater than $0.1^{\circ} \mathrm{C}$.

The camera was positioned in a vertical stand $1 \mathrm{~m}$ away from the animal.

The images were processed in $3.0 \mathrm{GHz}$ Pentium IV computer coupled to a PCMCIA card. Through the specific program ThermaCAMTM Researcher, FLIR Systems (Sweden), the images were recorded up to seven frames per second, throughout the time of the pre-injection, followed during injection and for 30 minutes under the same environmental conditions already described. All images were represented by infrared thermograms in a video monitor and recorded on CD Rom for analysis with the software.

The images were plotted using thermal amplitude (range) between $32.9^{\circ} \mathrm{C}$ and $19.2^{\circ} \mathrm{C}$, average (level) of $30^{\circ} \mathrm{C}$ and colorimetric scale (palette) continuous "RAINBOW900" in which white, red, yellow, green, blue and black represented, respectively, a sliding scale of temperature areas, equally distributed in the range from warmer to colder. This was maintained until the end of the experiment.

For purposes of the thermodynamic study, the results of the filming were pre-analyzed in graphical form as follows:

\section{1. $Y$ axis - two variables:}

The maximum temperature was captured inside the acrylic cage where the mouse was. Once the animal moved unrestrictedly in the cage, the points of highest temperature would be mapped, corresponding to the central compartment, i.e., eye and ear.

The average temperature, captured in the entire area of the experiment, that is, the mouse inside the cage. It had the purpose of demonstrating the phenomena intrinsic to the animal that were significantly influenced by this semi-closed system of heat exchange.

\section{X-axis - time in minutes.}

After the procedure, the animals were transferred to appropriate cages and observed until recovery. The cages were identified according to the group, being distributed five animals in each cage. Free access to water was provided, and power was restored after 12 hours. We observed the clinical status of animals on a daily basis, evaluating motor activity, behavior and 
any signs suggestive of disease. After the experiment, animals were sacrificed with a lethal dose of inhaled ether.

For the analysis of thermal changes, Gaussian attributes, independence and homogeneity of variances were tested.

When the previous tests met the proposed criteria, we used univariate ANOVA and Tukey's parametric test to establish the significance of the results.

In all tests, the range stood 0.05 or $5 \%(p<0.05)$ as a rejection of the null hypothesis. To this purpose, we used the specific program Statistica 5.1 .

Initially, the accuracy limits (PT) for measurements of temperature were calculated as twice the standard deviation of each set of observations of temperature $T(P T=2 s T)$ and intrinsic sensor error (Bias limit), BT $=0.001 \mathrm{~K}$ for each temperature point. Thus, the limit of precision in the measurement of $\mathrm{T}$ was estimated using the propagation equation proposed by Kline and McClintock quoted in Kline and Steele (1953):

$$
\mathrm{UT}=\sqrt{ } \mathrm{PT} 2+\mathrm{BT} 2
$$

$\mathrm{UT}=$ temperature measurement uncertainty; $\mathrm{PT}=$ limit precision, $\mathrm{BT}=$ intrinsic sensor error (bias limit)

As BT is much smaller than PT, the term is negligible in the presence of the equation. Consequently, the uncertainties of the temperatures for each point were computed as follows:

In the graphs of the results of this study, each point of temperature is represented as $\mathrm{T} \pm \mathrm{UT}$. The first law of Thermodynamics was applied to this model for a closed system in steady state.

The volume-control system was defined as the region between the central portion and periphery of the animal, that is, from the internal organs to the external skin surface. We adopted the measurement of a constant temperature and uniform heat distribution representative for this volume-control, which is the core temperature. We measured the heat loss from the mouse to the external environment, taking into account the mass and specific heat of the animal $\left(3.8 \mathrm{~kJ} / \mathrm{kg} .{ }^{\circ} \mathrm{C}\right)$, divided by total experiment time:

$$
\begin{aligned}
& \mathrm{Q}=\mathrm{m} . \mathrm{C} . \mathrm{DT} / \mathrm{Dt} \\
& \mathrm{Q}=\text { heat loss }[\mathrm{W}] ; \mathrm{m}=\text { animal mass }[\mathrm{kg}] ; \mathrm{c}=\text { specific heat of the animal } \\
& {\left[\mathrm{J} /\left(\mathrm{kg} .{ }^{\circ} \mathrm{C}\right)\right] ; \mathrm{DT}=\text { difference between the initial and final temperature }\left[{ }^{\circ} \mathrm{C}\right] ;} \\
& \mathrm{Dt}=\text { time interval of each experiment }[\mathrm{s}] .
\end{aligned}
$$

Thus, we determined the power (or rate of heat transfer) in each group. The results of the heat loss from the central compartment were expressed in Watts (Watts), that is, heat loss (Joule) per unit time (seconds).

\section{RESULTS}

Environmental variables (temperature, humidity and air velocity) remained stable throughout the procedure and no animal died during the experiment. The average temperature analysis captured the entire area of the experiment, that is, monitoring the mouse (the object of study) in the cage (environment), and was represented by variable $A$ in the $Y$ axis. That plotted the filming of the thermodynamic study and served to demonstrate the phenomena intrinsic to the animal that were significantly influenced by the heat exchange system. Due to the constant movement of the animal, it was not always possible to have an orthogonal angle with the thermal imager, which recorded the highest temperature points, the eyes and ears. The graphic analysis of variable $B$ showed an oscillating pattern of uptake of the maximum temperature in the thermogram, eye and ear (Figure 1 variable black line B Y-axis). In this study, eyes and ears were identified as points of higher temperature, corresponding to the central compartment.

\section{GRAPHICAL REPRESENTATION OF THE THERMOGRAMS}

\section{Group C or Control Group}

Y-axis: variable $(A)$ graphically represents the average temperature of the thermal changes of the rat in the cage; variable (B) represents the maximum temperature of eyes and ears of the animal.

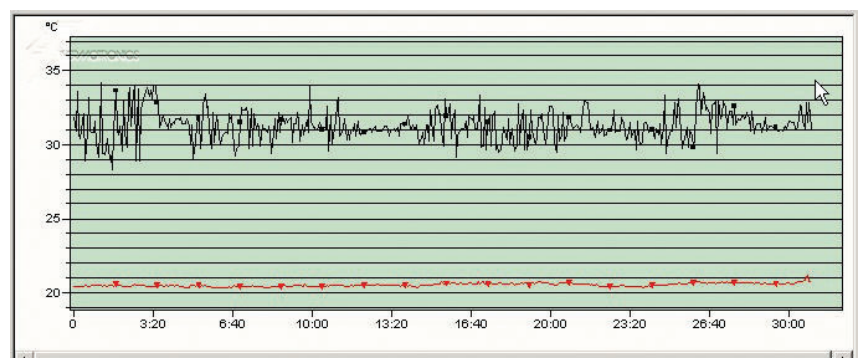

Figure 1 - Graphical Analysis: Animal Footage of Group C.

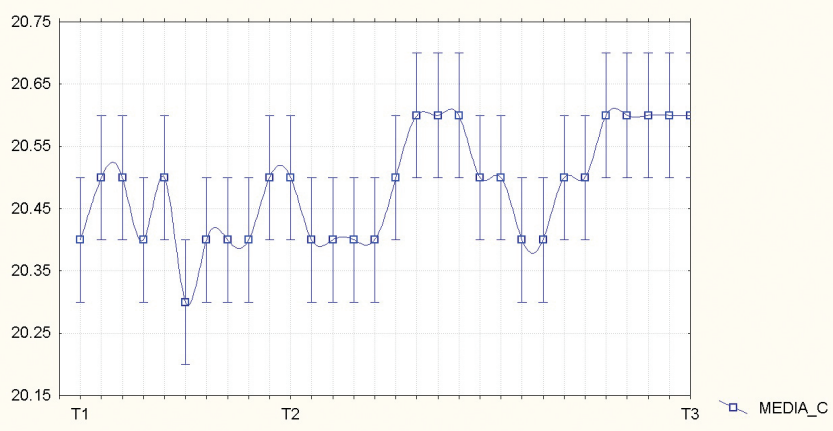

Graphic 1 - Mean \pm UT Temperature Average of Animals in Group C.

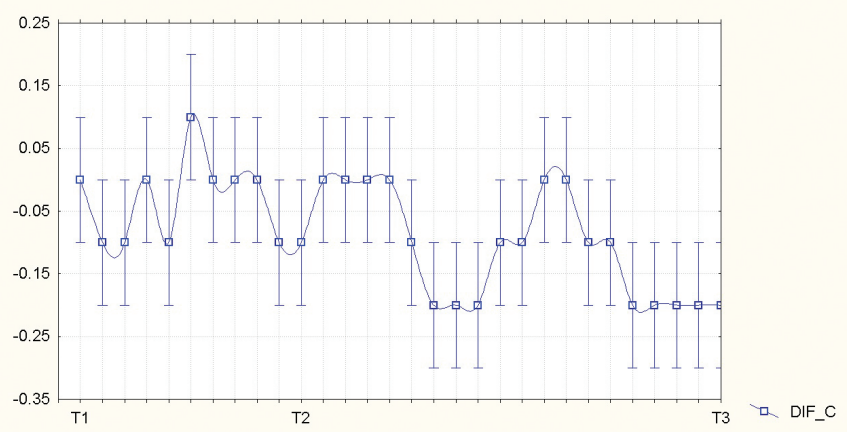

Graphic 2 - Mean \pm UT Temperature Rise $\left({ }^{\circ} \mathrm{C}\right)$ in Group C. 


\section{Group B or Group Bupivacaine}

In group $B$, there was a temperature rise of about $1^{\circ} \mathrm{C}, 5 \mathrm{~min}$ utes after the intraperitoneal injection of bupivacaine, which persisted until 18 minutes after the experiment, decreasing after 20 minutes, according to Figure 2. Differences in vasomotor response in Group B compared to $\mathrm{C}$ were observed.

The graphic analysis of the variable, which represents the average temperature of bupivacaine group, showed increase in average temperature in the cage (dashed red Figure 2), evidence that the use of bupivacaine altered the physiology of the animal, promoting change in temperature average of all rat-cage.

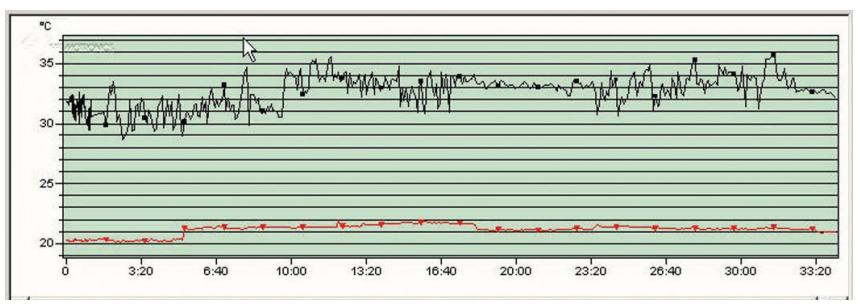

Figure 2 - Graphical Analysis of Group B Thermogram (bupivacaine).

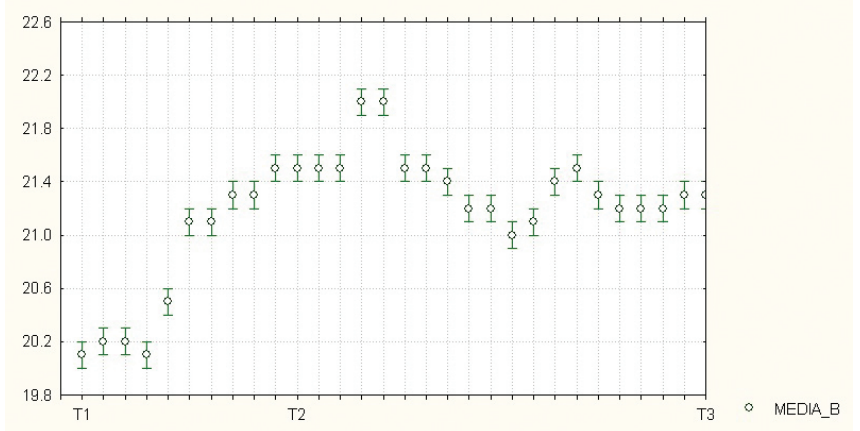

Graphic 3 - Mean \pm UT Average Temperature of Animals in Group B.

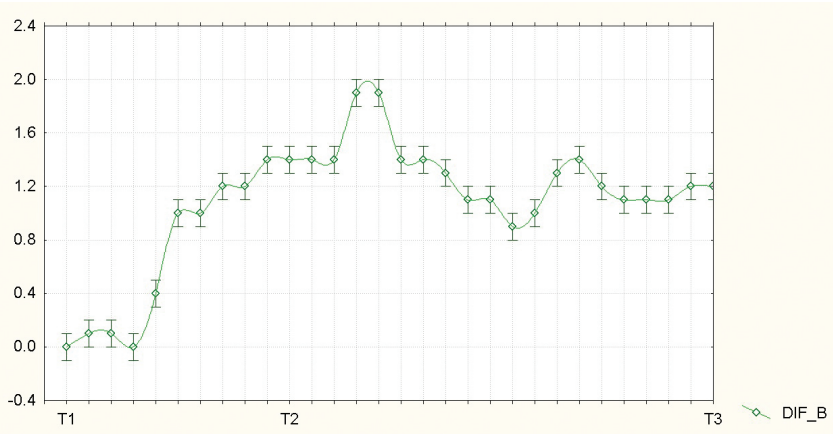

Graphic 4 - Mean \pm UT Temperature Rise $\left({ }^{\circ} \mathrm{C}\right)$ in Group B.

\section{Group L or Group Levobupivacaine}

The results of graphic analysis showed no difference between groups Levobupivacaine and Control. The average tempera- ture of the cage remained stable throughout the experiment (variable $A$, red line on the $Y$ axis).

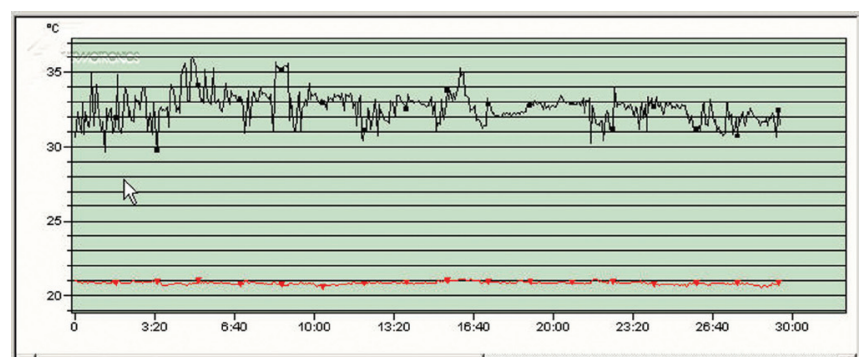

Figure 3 - Graphical Analysis of Group L.

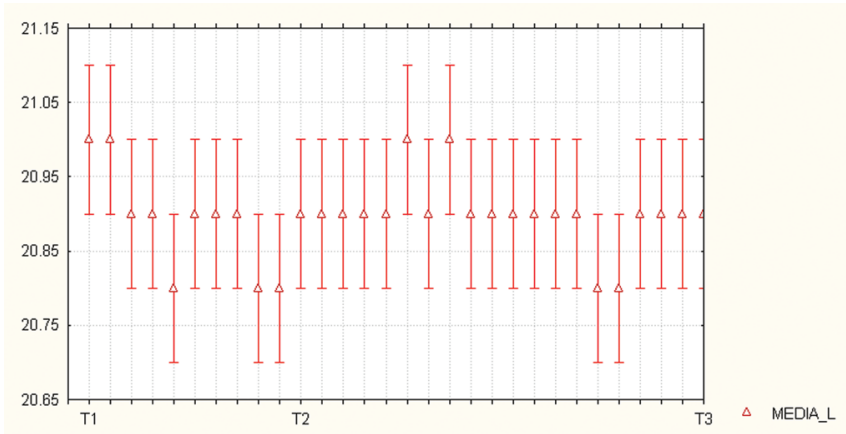

Graphic 5 - Mean \pm UT Average Temperature of Animals in Group L.

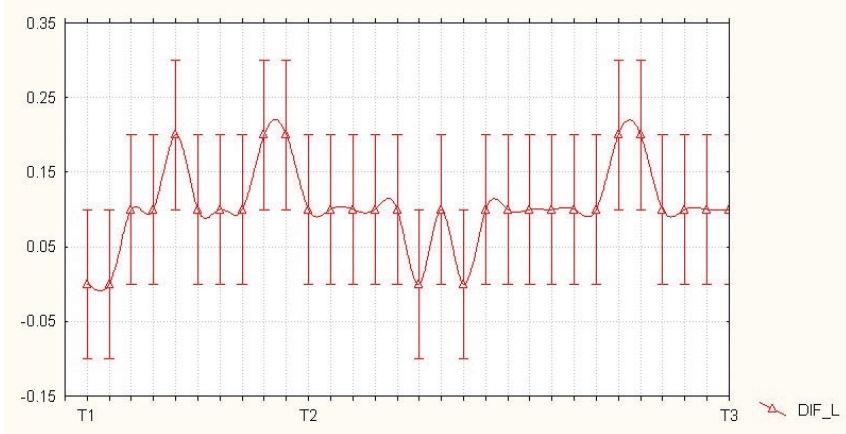

Graphic 6 - Average Temperature Rise of \pm UT $\left({ }^{\circ} \mathrm{C}\right)$ Group L.

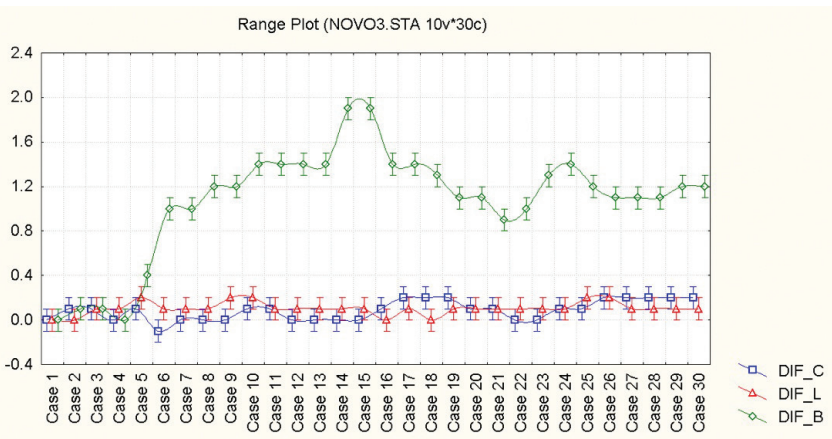

Graphic 7 - Average Temperatures Differences of Averages in All Groups. 


\section{Thermograms}

After the identification of the graphical difference, the thermograms specific for the analysis were searched in the database. Here were shown the T1 (intraperitoneal injection), T2 (10 minutes after the initial thermogram) and T3 (30 minutes after injection) in relation to groups $\mathrm{C}, \mathrm{B}$ and $\mathrm{L}$.

The initial thermograms T1 were homogeneous in the three groups, with no temperature change in the opening minutes in either group.

There was no difference between groups $C$ and $L$ at all times, as shown in Figures 4, 5 and 6.

Therefore, thermograms T1, T2 and T3 are represented only once, without reference to group $\mathrm{C}$ or $\mathrm{L}$.

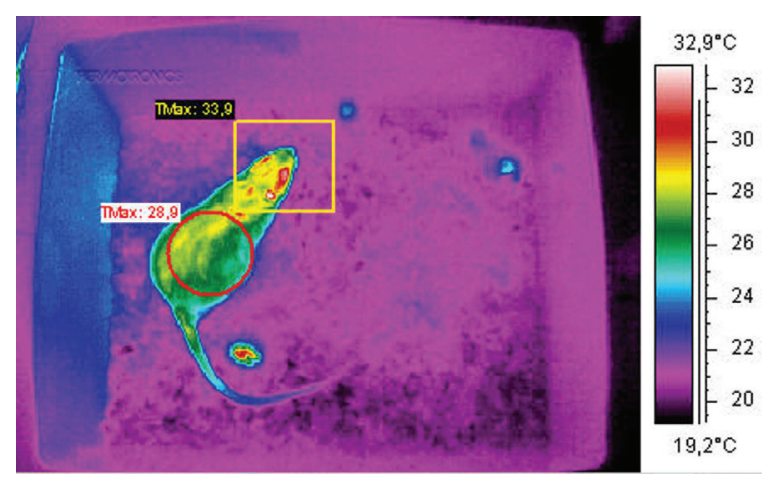

Figure 4 - T1 - Initial Thermogram of Groups: Control and Levobupivacaine.

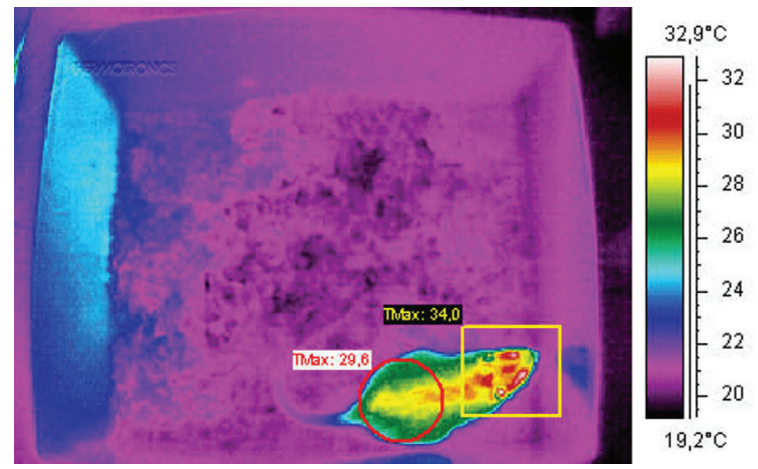

Figure 5 - T2 - Thermogram 10 Minutes After Injection, Groups C and L.
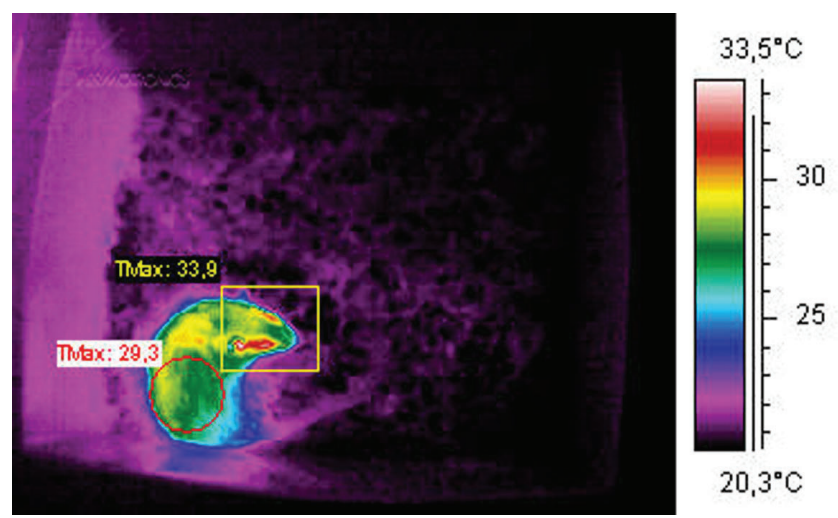

Figure 6 - T3 - Thermogram 30 Minutes After Intraperitoneal Injection, Groups $\mathrm{C}$ and L.
Group B was different in relation to Groups C and L in T2 and T3. The increase of temperature in the region of eyes and ears in T2 and T3 is noteworthy.

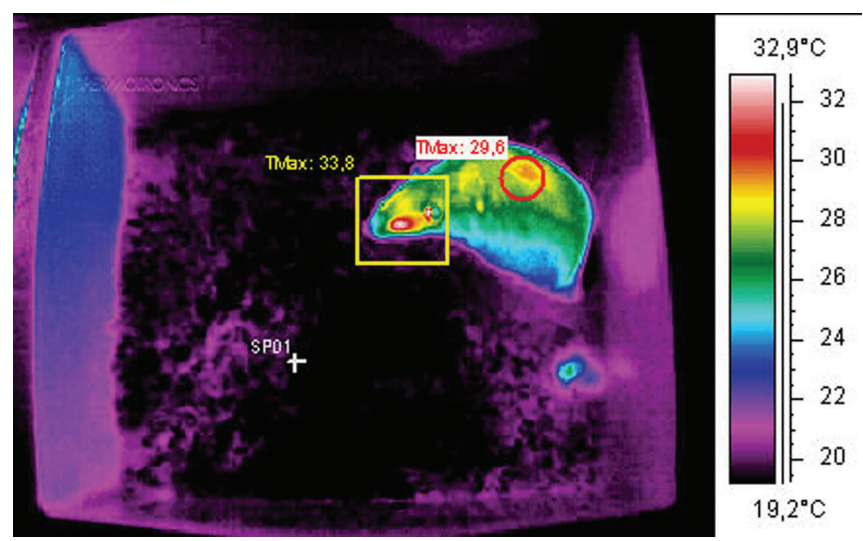

Figure 7 - T1 - Group B or Bupivacaine.

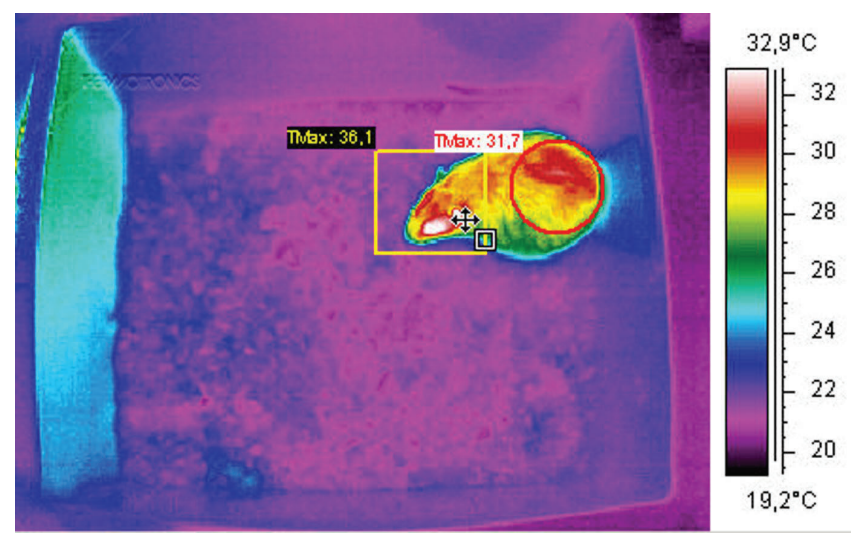

Figure 8 - T2 - Group B.

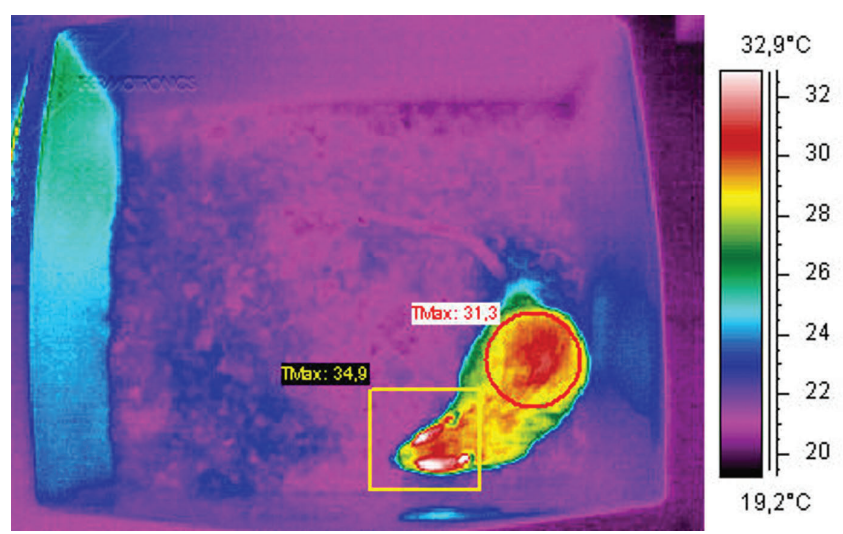

Figure 9 - T3 - Group B.

There was no statistical difference between the values found in the temperature difference between T1 and T3 in the control group and levobupivacaine.

There was statistical difference between the values found in the temperature difference between T1 and T3 in the control groups compared to levobupivacaine and bupivacaine, as the Chart 1. 
The values expressed in $\mathrm{W}$, that is, $\mathrm{J} / \mathrm{s}$ are obtained by dividing the values from the table above for 1,800 seconds ( 30 minutes of experiment).

Chart 1 - Rate of Heat Transfer Between Groups.

\begin{tabular}{ll}
\hline Control group & $91.8 \pm 96.34 \mathrm{~J}$ \\
Levobupivacaine & $113.87 \pm 61.2 \mathrm{~J}$ \\
Bupivacaine & $1,182.81 \pm 541.36 \mathrm{~J} *$ \\
\hline
\end{tabular}

* Statistically significant [corpo de legenda]

\section{DISCUSSION}

Through this model, it was found that the group intoxicated with levobupivacaine had a behavior similar to control. In the group intoxicated with bupivacaine, there was difference in results from the mean temperature of the cage+rat complex, and increase of temperature of the animals, mainly around the eyes and ears, and also the back. This phenomenon could be explained by vasoconstriction, with diversion of blood circulation from the periphery to the heart-lung-brain. It explained, thus, hyperthermia found in eye-ear region. Another plausible hypothesis is that there are muscular contractions, to the extent that there is intraperitoneal absorption of AL.

In general, studies on the effects of vasomotor symptoms of local anesthetic toxicity are controversial and are influenced by several variables. The effects of bupivacaine on the peripheral vasculature are also controversial, being described both vasodilation and vasoconstriction.

The vasodilatory effects of nicardipine and phentolamine increase the cumulative dose, but do not affect the plasma level to trigger seizures induced by bupivacaine. Bernards et al. ${ }^{11}$ observed that rats protected with nicardipine and phentolamine required, respectively: $7.6 \pm 1.5$ and $8.1 \pm 1.1$ mg. kg ${ }^{-1}$ compared to control $5.8 \pm 1.5 \mathrm{mg} \cdot \mathrm{kg}^{-1}$ of bupivacaine infused continuously to provoke convulsion. At the time of seizure, brain and plasma levels were similar in terms of statistics, which support the thesis that the vasoactive phenomena interfere in the dynamics of the distribution of $\mathrm{AL}$ in the model of acute toxicity by intravenous infusion.

A protective effect of propofol and sevoflurane in models of acute toxicity by bupivacaine in rats was observed, suggesting that these mechanisms of protection are not sufficiently clarified and point to a central action of bupivacaine on the GABAergic system ${ }^{12}$.

Regarding the assessment of the influence of clonidine on bupivacaine EV toxicity, there was an effect due to the antiarrhythmic properties of a2 agonist. Considering significant the acute intoxication with epidural bupivacaine with accidental intravascular injection in bolus, there is a plausible association with the use of clonidine in the block instead of opioids. The nicardipine has also shown to be protective in acute venous intoxication, having postulated a mechanism of protection by inhibition of calcium channels and reduction of interactions with bupivacaine ${ }^{15}$.

A study of bupivacaine toxicity in pigs found that the addition of epinephrine significantly increases peripheral vasoconstriction and toxicity, being necessary the administration of a lower dose to seizures and cardiac arrest ${ }^{10}$.

The monitoring parameters such as mean arterial pressure, electroencephalography, and electrocardiography were not part of this protocol, in order to avoid contact from the animal with the monitoring equipment that could influence the results of this research.

When a group intends to study the vasomotor state of a living being, there must be adequate preparation of the animals, ability to control the atmospheric phenomena in the laboratory, and experimental use of high precision equipment. These items have been carefully examined and checked for the establishment of experimental validation of this model.

In this study, the animals were kept in a 12-hour fasting period before the experiment, since some studies have revealed that part of the energy gained from food is released as heat ${ }^{16}$.

After a meal, the body surface temperature may increase from $1^{\circ} \mathrm{C}$ to $2^{\circ} \mathrm{C}$, reaching its peak 30 minutes after the meal ${ }^{17}$.

The animals were kept hydrated ad libitum, as water balance is another important factor that affects surface temperature. If the body's water content is reduced, its thermal conductivity will also be reduced ${ }^{18}$.

The temperature of the body depends on its blood flow when the body produces excessive heat during intense exercise, and rectal temperature may temporarily increase to 1.3 up to $3^{\circ} \mathrm{C}$ more than the normal core temperature ${ }^{19}$. So it was avoided that the animals increased their physical and metabolic activity before the experiment. They were picked up gently by the back to prevent escapes or higher stress before the intraperitoneal injection. In physical activity, heat production may increase 10 to 20 times, increasing muscle temperature higher than the core temperature ${ }^{20}$. The release of hormones under stress such as adrenaline can cause cutaneous vasoconstriction and reduce skin temperature, which leads to a heterogeneity in the initial temperature in mice of the same group, if precautions are not taken ${ }^{21}$.

Therefore, this study found that the vasomotor effect of the acute toxicity of levobupivacaine was similar to the control group with saline, through macroscopic studies by digital infrared footage.

There were vasomotor alterations with bupivacaine intoxication (vasoconstriction), more than in relation to the control group and in relation to the Levobupivacaine Group. 